\title{
Queratinas de plumaje de pollo y lana de oveja: Comparativa de sus masas moleculares mediante electroforesis en gel
}

Fernández-d'Arlas Bidegain, B.

INAMAT-Institute for Advanced Materials. Universidad Pública de Navarra (UPNA). Pamplona. Navarra. España.

\section{PALABRAS CLAVE ADICIONALES}

\section{Queratina.}

Lana.

Pluma.

Pollo.

Masa molecular.

Electroforesis.

\author{
ADDITIONAL KEYWORDS \\ Keratine. \\ Wool. \\ Feather. \\ Molecular weight. \\ Electrophoresis.
}

\section{RESUMEN}

El plumaje de pollo y la lana de oveja son actualmente considerados residuos agroindustriales de difíil deshecho. Es por ello que la búsqueda de aplicaciones para estos materiales es interesante desde el punto de vista de la Economía Circular. El conocimiento de las propiedades y características de sus queratinas, el principal constituyente de estos materiales, puede ayudar en el diseño de sus aplicaciones finales. Este trabajo expone los resultados del análisis de la masa molecular de las queratinas de la lana de oveja lacha (Ovis aries) y el pollo de consumo (Gallus domesticus) obtenidas mediante extracción reductiva con tioglicolato sódico en medio alcalino. El análisis llevado a cabo mediante electroforesis en gel de poliacrilamida indica que la queratina de la lana posee una masa molecular con fracciones de 56 , $47,14,10$ y $8 \mathrm{KDa}$, mayor en promedio que la queratina del plumaje de pollo, únicamente con fracciones de menor masa molecular comprendidas entre 8-6 KDa.

\section{Keratins from chicken feathers and sheep wool: Comparison of their molecular weight by gel electrophoresis}

SUMMARY

Chicken feathers and sheep wool are at present considered two major agro-industrial residues which are hard to be disposed. Therefore finding applications for these residues is interesting from the viewpoint of Circular Economy. The knowledge of the properties and characteristics of keratins, which are the main constituent of these materials, can help designing final applications for them. This report presents the results of the comparative analysis of the molecular weights of keratins from Lachas heep (Ovis aries) and consumer chicken (Gallus domesticus) obtained by reductive extraction with sodium tioglycolate inalkalyne media. The analysis was carried out trough polyacrylamide gel electrophoresis and showed that wool keratin has molecular weightfractions of $56,47,14,10$ y $8 \mathrm{KDa}$, higher on average than those of the feathers, only with lower molecular weight fractions, in the range of 8-6 KDa.

INFORMATION

Cronología del artículo.

Recibido/Received: 19.07.2017

Aceptado/Accepted: 08.09.2019

On-line: 15.07.2019

Correspondencia a los autores/Contact e-mail:

borja.fernandezdarlas@unavarra.es

\section{INTRODUCCIÓN}

En España en torno a 36.000 toneladas de lana son desechadas anualmente debido a su baja calidad para textiles (Marsal et al. 2009). El destino de las plumas de pollo es similar, con en torno a 100.000 toneladas producidas solo en España y más de 5 millones a nivel mundial (Poole et al., 2009). Por ello, la extracción de la queratina de estos subproductos con el objeto de desarrollar aplicaciones a partir de ellas es una vía interesante de revalorización, ya que la queratina tiene potencial de ser transformada en materia prima para biopelículas (Yamaguchi et al. 1996) y bioplásticos (Moore et al. 2005), fertilizantes (Jie et al. 2008 y Vega-Zavaleta et al. 2014), apósitos biocompatibles y bioabsorbibles o excipientes para fármacos (Rouse y Dyke, 2010, y Saravanan et al. 2013). Conocer la masa molecular que las cadenas de queratinas son esencial a la hora de enfocar su uso en distintas aplicaciones por lo que en este trabajo se pretende dilucidar las diferen- 
cias de masas moleculares de las queratinas extraídas de dos deshechos agroindustriales paradigmáticos: el plumaje de pollo (Gallus domesticus), empleado como fuente de carne, y la lana de oveja lacha cara negra (Ovis aries), empleada en la producción de quesos de pastor de tipo Idiazábal.

\section{MATERIAL Y MÉTODOS}

\section{MATERIAL BIOLÓGICO}

Las muestras de plumas de pollo de consumo ( $\mathrm{Ga}$ llus domesticus) fueron suministradas por Avícola Mélida S.L, de Mélida, Navarra, España. Las muestras de lana de oveja lacha caranegra (eusk.: Latxa mutturbeltz), figura 1a, fueron suministradas por la quesería baztanesa Etxetxipia (Pron.: "echechipia"; eusk.: Etxetxipia Gaztategia). La lana fue esquilada en Mayo de 2016 y provenía de especímenes adultos. La quesería está situada en el municipio de Elizondo, en el Valle de Baztán, en el norte de Navarra, España. Los animales fueron tomados al azar de manera que los factores incontrolados que afectan los contenidos y la calidad de estos materiales, como es el caso de los metabolitos sanguíneos, fueron aleatorizados (Amarilho-Silveira et al. 2017).

\section{EXTRACCIÓN DE QUERATINA}

Las muestras fueron lavadas y desengrasadas sumergiendo $100 \mathrm{~g}$ de material seco en $15 \mathrm{~L}$ de agua conteniendo $20 \mathrm{~g}$ de jabón (Buga, Bunzl Spain S.A) con $5-15 \%$ de tensioactivos aniónicos y $<5 \%$ tensioactivos no-iónicos. Tras $\sim 20 \mathrm{~h}$ a remojo fueron aclaradas con un caudal constante de agua, hasta retirar el jabón residual. Posteriormente las fibras fueron escurridas y secadas en horno a $50{ }^{\circ} \mathrm{C}$ durante $\sim 24 \mathrm{~h}$. Las muestras fueron molidas en un molino (Retsch) con una malla de $\sim 1 \mathrm{~mm}$. Para la extracción de la queratina $\sim 0,6 \mathrm{~g}$ de material fueron suspendidos en $15 \mathrm{~mL}$ de ácido tioglicólico (Panreac, España) 0,5 N neutralizado con $\mathrm{NaOH}$ hasta $\mathrm{pH}=11$. Las mezclas fueron agitadas magnéticamente en baño de aceite a $50{ }^{\circ} \mathrm{C}$ durante $2 \mathrm{~h}$. Después las dispersiones fueron filtradas con papel de filtro y ayuda de vacío en matraz kitasatos. La queratina se precipitó a partir del filtrado adicionando HCL 3N hasta $\mathrm{pH} \sim 4$. Posteriormente las queratinas fue insertada en membranas celulósicas semipermeables (Viscofán, España) y dializada en un tanque de 25 L durante 4 días, renovando el agua osmotizadaconstantemente. Finalmente fueron dializadas frente a agua desionizada de 16 M $\Omega$ (Wasserlab, Pamplona, España). Las queratinas lavadas fueron secadas en condiciones ambientales en campana con flujo de aire constante. La queratina de lana resulto muy blanca mientras que la de pluma presentaba tonalidades amarillentas.

\section{MedidA DE MASAS MOLECULARES DE LAS QUERATINAS}

La masa molecular de las queratinas de lana y pluma fue analizada mediante electroforesis en gel de poliacrilamida/bisacrilamida (Acri/Bis), compuesto de una fase de concentración (Tris- $\mathrm{HCl}, 0,125 \mathrm{M}, \mathrm{pH}$ = 6,6; 4\% Acril/Bis) y una de separación (Tris- $\mathrm{HCl}$, 0,375 M, pH = 8,8; 18\% Acril/Bis), donde Tris- $\mathrm{HCl}$ es tris(hidroximetil)aminometano (Panreac) neutralizado con HClhasta el pH indicado.Los geles contenían 0,2\% en masa de dodecil sulfato sódico (SDS) como agente desnaturalizante. La muestras fueron tratadas en un tampón de electroforesis consistente en Tris- $\mathrm{HCl} 20$ $\mathrm{mM}, \mathrm{pH}=8,8 ; 2 \%$ SDS, urea 3,6 M, 15\% de glicerol y $5 \%$ de $\beta$-mercaptoetanol (Panreac). Se han pesado entre 2-4 mg de queratinas de lana y pluma y diluido en el tampón hasta una concentración de $\sim 4 \mathrm{mg} \cdot \mathrm{mL}^{-1}$. A las muestras se les ha añadido azul de bromofenol hasta concentración del 0,05\% masa. Las muestras han sido incubadas a $97^{\circ} \mathrm{C}$ durante 3 min para minimizar la actividad de las posibles proteasas presentes, se han sonicado en baño 10 min y se han centrifugado durante 10 s. Se han tomado $7 \mu \mathrm{L}$ de muestra y patrón (ProteinMarker VI, 10 - 245KDa, Panreac) del fondo del vial y se han corrido a 200-240 V en cuba electroforética (Miniprotean, Biorad) con tampón de marcha Tris-glicinaSDS, $0,375 \mathrm{M}, \mathrm{pH}=8,8$, hasta llegar el bromofenol hasta el fondo del gel.

\section{ANÁLISIS DE ELECTROFEROGRAMAS}

Los electroferogramas obtenidos mediante electroforesis han sido capturados con un escáner de sobremesa y analizados con el programa en abierto ImageJ. Para obtener una recta de calibrado (masa molecular vs. distancia recorrida) a partir del patrón de masas moleculares se ha estudiado la distancia (en píxeles) de cada una de las bandas correspondientes a las distintas proteínas del patrón, tomando como punto de partida la interface entre el gel de concentración y el gel separador.

\section{RESULTADOS Y DISCUSIÓN}

\section{NATURALEZA DE LAS MUESTRAS}

Para la extracción de las queratinas se ha empleado el método reductivo descrito por Goddard y Michaelis (1934) mediante el cual tioglicolato de ( $\left.\mathrm{HS}-\mathrm{CH}_{2}-\mathrm{COO}^{-}\right)$ rompe los enlaces bisulfuro de la queratina (figura $\mathbf{1 b}$ ) logrando solubilizar las macromoléculas. El medio básico $(\mathrm{pH}=11)$, además ayuda a desprotonar algunos residuos cargados positivamente, como las lisinas, minimizando los enlaces intermoleculares salinos. Cabe mencionar que las muestras de lana y pluma molidas presentan densidades muy diferentes. En la figura 1c se muestran botellas conteniendo $11 \mathrm{~g}$ de lana y pluma sin moler y molida. Puede observarse que los materiales de partida (i.e., sin moler) ocupan volúmenes semejantes mientras que comparando los materiales molidos, la pluma ocupa entorno al doble de volumen que la lana. Ello puede deberse a la estructura "pseudo-fractal" (López-Albors et al. 1999) de las plumas (figura 1d) que evita un empaquetamiento efectivo de las fibras molidas.

\section{MASA MOLECULAR DE LAS QUERATINAS}

La figura 2a muestra los geles con el patrón y las proteínas extraídas de pluma y lana. La figura $\mathbf{2 b}$ muestra el electroferograma del patrón y la figura 2c la recta de calibrado (distancia recorrida-masa molecular). La figura 3d muestra los electroferogramas de las proteínas de pluma y lana en función de la masa molecular, representación para la cual se ha empleado el calibrado de la figura 2c. Estudiando las figuras $\mathbf{2 a}$, $\mathbf{d}$ se puede observar que el extracto de lana presenta bandas 

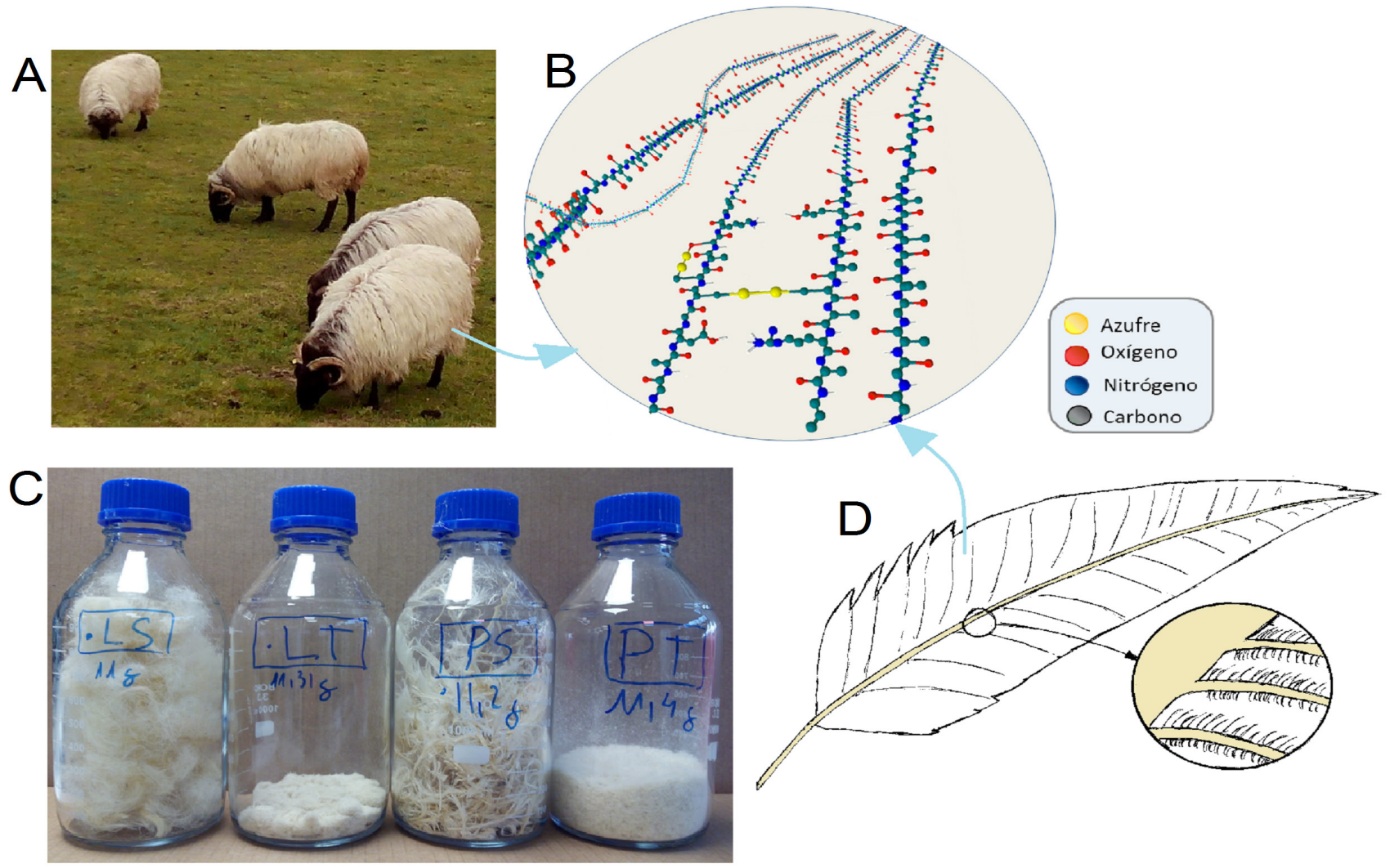

Figura 1. Características de las fuentes de queratina. Ovejas lacha caranegra pastando en Elizondo, Navarra, cuya lana ha sido empleada para la extracción de queratina en este estudio (A); Esquema de la estructura general de la queratina según Gacén-Guillén (1964) (B);Botes conteniendo 11 g de lana, lana triturada, pluma y pluma triturada, de izquierdaa derecha, respectivamente (C); Detalle de la estructura "pseudo-fractal" de una pluma. (Characteristic of thekeratinsources. Lacha sheep pasturing in Elizondo, Spain, whose wool has been used forkeratin extraction in thepresent report (A); Scheme of the general keratin structure according to Gacén-Guillén (1964) (B); Vials containing $11 \mathrm{~g}$ of wool, milled wool, feathers and milled feathers, from left to right, respectively (C); Detail of the "pseudo-fractal" structure of a feather (D))

claras de las denominadas queratinas- $\alpha$ o queratinas de filamentos intermedios, de alta masa molecular, con fracciones de 47 y $56 \mathrm{KDa}$. El extracto de la pluma, sin embargo, no presenta bandas intensas en esta región de alta masa molecular. La práctica ausencia de bandas en esta región para queratinas de pluma también ha sido reportada por Moore et al. (2006). Estos resultados están de acuerdo con numerosas investigaciones que ponen de manifiesto la pérdida de importancia de las queratinas- $\alpha$ en las plumas avícolas a lo largo de la evolución y domesticación (Greenwold et al. 2014).

Análisis de la masa molecular de queratinas de oveja lacha llevados a cabo mediante dispersión estática de luz de dispersiones acuosas, han dado masas moleculares promedio en peso muy superiores (Fernández-d'Arlas, et al. 2016). Este trabajo también puso de manifiesto que las queratinas son termodinámicamente inestables en agua, tendiendo a coalescer, por lo que aquellos resultados pueden interpretarse por la presencia de agregados. También es cierto que en las interfaces de los geles (figura 2a) pueden apreciarse acumulación proteica, por lo que no es descartable la presencia en estas muestras de fracciones de muy alta masa molecular (> $100 \mathrm{KDa})$.

También, la queratina de la lana presenta una banda muy intensa centrada en $13,9 \mathrm{KDa}$, mientras que la queratina de pluma presenta en esa región un halo disperso, probablemente debido a la presencia de fracciones polidispersas de masa moleculares no bien definida. En el caso de la lana estas bandas pueden relacionarse con las proteínas asocidas a queratinas (KAPs, keratin associated proteins) (Fujikawa et al. 2013). La queratina de pluma si presenta picos bandas claras centradas en 7,9 y 6,1 KDa. En esta última región ( $6 \mathrm{KDa})$ la queratina de lana no presenta ninguna banda lo cual puede atribuirse la exclusividad de algunas queratinas del tipo $\beta$ a los reptiles y aves (Greenwoldet al. 2014). A su vez, las bandas de 47 y $57 \mathrm{KDa}$ presentes de manera relevante en las queratinas de la lana lacha pueden estar relacionadas con las queratinas a de interfilamentosas de tipo I (acídicas) y tipo II (básicas), respectivamente, características de muchos mamíferos (Fuchs y Marchuk, 1983), y también presentes en pelos humanos (Reichl y Goymann, 2011), lanas y cornamentas de otras ovejas como la merino (Zoccola et al. 2009).

\section{CONCLUSIÓN}

La distribución de masas moleculares de las proteínas de lana se caracteriza por la presencia de queratinas con fracciones de alta masa molecular (47-57 KDa) atribuibles a la alta proporción de queratinas- $\alpha$ de tipo I y II característica de los mamíferos. Las proteínas de pluma presenta únicamente fracciones de baja masa 


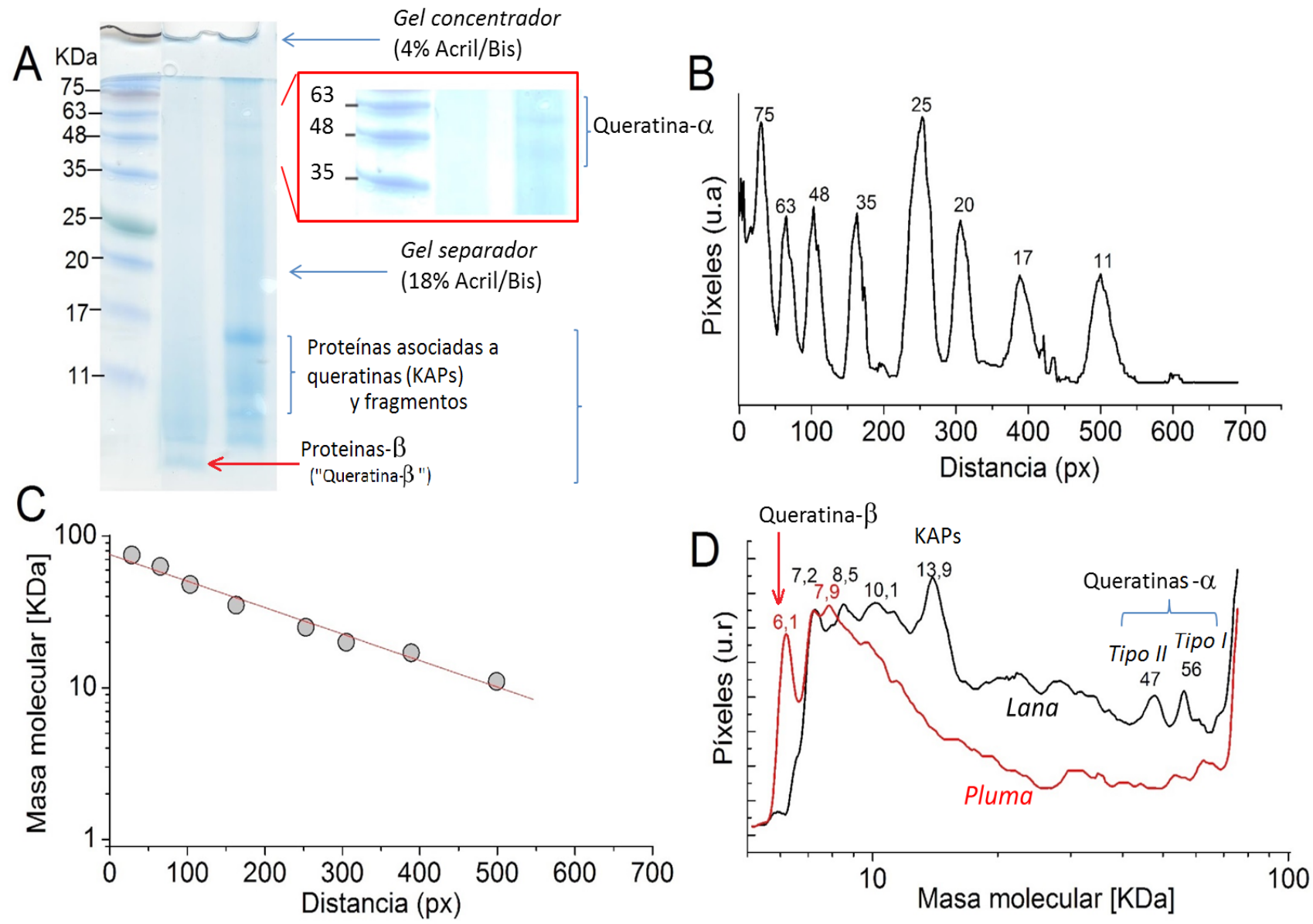

Figura 2. Análisis electroforético de las proteinass de lana y pluma. Electroferogramas del patrón, y proteínas de pluma y lana, de izquierda a derecha, respectivamente, junto con detalle de la zona entre 30-70 KDa (A); Perfil de número de píxeles oscuros (unidades arbitrarias) frente a la distancia recorrida (en píxeles) para el patrón de proteínas (B); Recta de calibrado, $\log ($ Masa) vs.distancia $(p x),(R 2=0,977)(C)$; Perfiles de número de píxeles oscuros (unidades relativas) frente a la masa molecular, aplicando el calibrado de " $C^{\prime \prime}$, (D) (Electrophoretic analysis of the wool and feather proteins. Electropherograms of the protein marker, feather and wool proteins, from left to right, respectively (A); Profile of number of pixels (arbitrary units) against the run distance (in pixels) for the protein marker (B); Calibrating curveof log(Weight) vs. distance (px), (R2 $=0,977)(C)$; Profiles of the dark pixel number (relative units) against the molecular weight, by applying the calibration curve of "C", (D)).

molecular (6-8 KDa) siendo atribuidas las fracciones de menor masa molecular ( 6 KDa) a las denominadas queratinas- $\beta$ exclusivas de las aves y reptiles, tal y como se reporta en bibliografía (Greenwoldet al. 2014).

El conocimiento de las masas moleculares de las distintas fuentes principales de queratina permitirá profundizar en el conocimiento de la relación estructura-propiedades de los materiales desarrollados a partir de estas y así desarrollar materiales funcionales a medida en los campos de los plásticos o biomedicina, por ejemplo.

\section{AGRADECIMIENTOS}

El autor agradece la financiación de la Obra Social la Caixa y Fundación Caja Navarra en el marco del programa de la UPNA de "Captación de Talento" para desarrollar la presente investigación, así como al INAMAT por facilitarme el acceso a instalaciones y diversa instrumentación. El autor también agradece a Maria JesúsLarreche, de Etxetxipia, y al personal de la avícola Mélida, por el suministro de lana y plumaje, respectivamente, y asimismo a Viscofán S.A., por el la generosa aportación de membranas celulósicas semipermeables, óptimas para la diálisis.

\section{BIBLIOGRAFÍA}

Amarilho-Silveira, F; Vicente, SV; Lemes, JS; Del Pino, FAB.; Esteves, RM; Halfen, J; Tâmara, JQ; Da Vara, CC; Dionello, NJL; Mattei, P 2017 "Relação entre os níveis de metabólitos sanguíneos e a qualidade de Iã". Archivos de Zootecnia, vol. 66, no. 253, pp. 137-140.

Fernández-d'Arlas, B, Peña-Rodríguez, C, Eceiza, A 2016 "Extracción de la queratina de la lana de oveja "latxa" ." Revista lberoamericana de Polímeros, vol. 17, no. 3, pp. 110-121.

Fuchs, E\& Marchuk, D 1983 “Type I and Type II Keratins Have Evolved from Lower Eukaryotes to Form the Epidermal Intermediate Filaments in Mammalian Skin" Proceedings of the National Academy of Sciences of the United States of America, vol. 80, no. 19, pp. 5857-5861.

Fujikawa, H, Fujimoto, A, Faroog, M, Ito, M, \& Shimomura Y 2013 "Characterization of the Human Hair Keratin-Associated Protein 2 (KRTAP2) Gene Family". Journal of Investigative Dermatologyol. 133 , pp. 2780-2782.

Gacén-Guillen, J 1969 "Aspectos químicos del blanqueo de la lana con peróxido de hidrógeno: Modificación química de la queratina" Boletín del Instituto de Investigación Textil y de Cooperación Industrial, no. 39, pp. 43-70. 
Goddard, DR\&Michaelis, L 1934 "A study on keratin", Journal of Biological Chemistry, vol. 106, pp. 605-614.

Greenwold, M, Bao, W, Jarvis, ED, Hu, H, Li, C, Gilbert, MTP, Zhang, G\& Sawyer, RH 2014 "Dynamic evolution of the alpha ( $\alpha$ ) and beta $(\beta)$ keratins has accompanied integument diversification and the adaptation of birds into novel lifestyles" BMC Evolutionary Biology, vol. 14, pp. 249-265.

Jie, M, Raza, W, Xu, Y\& Shen, Q 2008 "Preparation and optimization of aminoacid chelated micronutrient fertilizer by hydrolyzation of chicken waste feathers and the effects on growth of rice", Journal of Plant Nutrition, vol. 31, no. 3, pp. 571-582.

López-Albors, O, Gil, F, Vázquez, JM, Latorre, R, Ramíre-Zarzosa, G, Orenes\& M, Moreno F 1999 "Revisión: nomenclatura e iconografía de las partes de la pluma y sus diferentes tipos" Anal. Vet., vol. 15, pp. 3-16.Marsal-Amenós, F, Morral-Romeu, E, Palet-Alsina, D 2009 "Puesta en valor de lanas y pieles de producción nacional. Ministerio de Medio Ambiente y Medio Rural y Marino", Dirección General de Recursos Agrícolas y Ganaderos. Subdirección General de productos Ganaderos, p.34 (España).

Moore, GRP, Martelli, SM, Andreo, PD, Gandolfo, CA, Machado, RFA, Bolzan, A \& Laurindo, JB 2005, "Obtenção de Biofilmes a partir de Queratina de Penas de Frango", Revista Materia, vol. 10, no. 1, pp. 8-13.

Moore, GRP, Martelli, SM, Gandolfo, CA, Pires, ATN \& Laurindo, JB 2006 "Queratina de penas de frango: extração, caracterização e obtenção de filmes", Ciênc. Tecnol. Aliment., Campinas, vol. 26, no. 2, pp. 421-427.
Poole, AJ, Church, JS \& Huson, MG 2009"EnvironmentallySustainableFibersfromRegeneratedProtein", Biomacromolecules, vol. 10, no. 1, pp. 1-8.

Rouse, JG \& Van Dyke, ME 2010 "A Review of Keratin-Based Biomaterials for Biomedical Applications", Materials, vol. 3, no.2, pp. 999-1014.

Saravanan, S, Sameera, DK, Moorthi, A \& Selvamurugan, N 2013 "Chitosan scaffolds containing chicken feather keratin nanoparticles for bone tissue engineering". International Journal of Biological Macromolecules, vol. 62, pp. 481- 486.

Stephan-Reichl, SL \& Müller-Goymann, CC 2011 "Keratin film made of human hair as a nail plate model for studying drug permeation" European Journal of Pharmaceutics and Biopharmaceutics, vol. 78, no. 3, pp. 432-440.

Yamauchi, K, Yamauchi, A, Kusunoki, T, Kohda, A \& Konishi, Y 1996, "Preparation of stable aqueous solution of keratins, and physiochemical and biodegradational properties of films", Journal of Biomedical Materials Research, vol. 31 , no. 4, pp. 439-444.

Vega-Zavaleta, L, Tellez-Monzón, L, Aravena, CN, Coral, MFC, Zavaleta, NV, Parker, RV \& Figueroa, LV 2014 "Aplicación de residuos sólidos hidrolizados del proceso de pelambre enzimático como fuente de aminoácidos libres en el crecimiento de plántulas de maíz", Saber y Hacer, vol. 2, no. 1, pp. 24-32.

Zoccola, M, Aluigi, A \&Tonin, C 2009 "Characterisation of keratin biomass from butchery and wool industry wastes" Journal of Structural Biology, vol. 938, no. 1-3, pp. 35-40. 